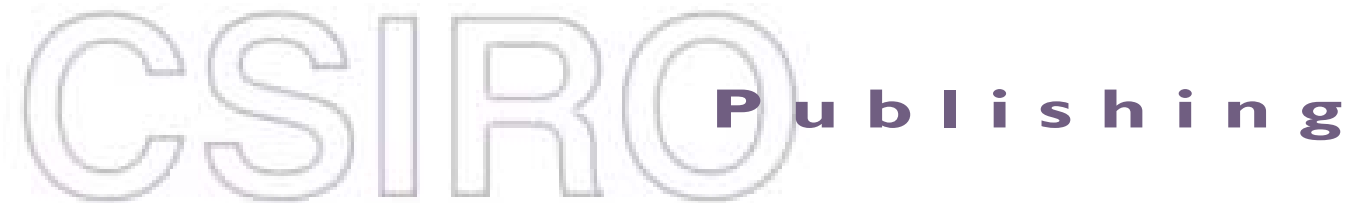

\section{Publications of the Astronomical Society of Australia}

Volume 19, 2002

(C) Astronomical Society of Australia 2002

An international journal of astronomy and astrophysics

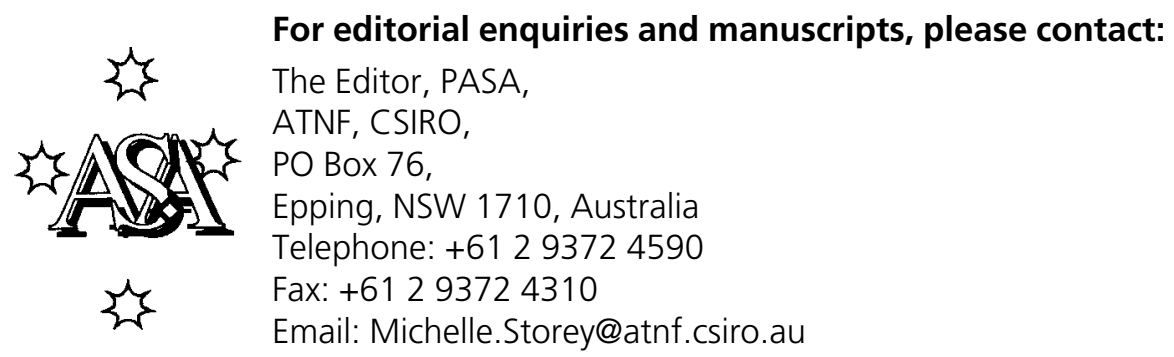

For general enquiries and subscriptions, please contact: CSIRO Publishing PO Box 1139 (150 Oxford St)

Collingwood, Vic. 3066, Australia

Telephone: +6139662 7666

Fax: +61 396627555

Email: publishing.pasa@csiro.au

C S I RO

PUBLISHING Published by CSIRO Publishing

for the Astronomical Society of Australia

www.publish.csiro.au/journals/pasa 


\title{
Single Dish Polarisation Calibration
}

\author{
Simon Johnston \\ School of Physics, University of Sydney, NSW 2006, Australia \\ simonj@physics.usyd.edu.au
}

Received 2001 August 1, accepted 2002 May 2

\begin{abstract}
Using the formalism of Hamaker, Bregman, \& Sault (1996), I derive a method for the polarisation calibration of observations made with a single radio telescope. This method is particularly appropriate for observations of pulsars, where the sign and magnitude of the circular polarisation are useful for understanding the emission processes at work. I apply the method to observations of PSR J1359-6038 made using the multibeam receiver on the Parkes radio telescope.
\end{abstract}

Keywords: polarisation — techniques: polarimetric — pulsars: general

\section{Introduction}

The polarisation properties of astronomical sources are interesting for a number of reasons. They can be used, for example, in determining the physical processes at work; thermal sources are not polarised, whereas non-thermal sources may have a significant degree of polarisation. The rotation measure of a source can be used to determine magnetic field strengths; for example pulsar rotation measures are used to derive magnetic maps of the Galaxy. However, the degree of polarisation in astronomical sources is often small, particularly circular polarisation, and so careful calibration is necessary before the results can be interpreted.

Recent papers on polarisation calibration of radio telescopes include those by Hamaker et al. (1996) and Britton (2000). The Hamaker et al. (1996) paper lays down the mathematical foundations which are subsequently used by Sault, Hamaker, \& Bregman (1996) to construct a method for instrumental calibration of a radio interferometer. Their method is routinely applied to data from the Australia Telescope Compact Array and incorporated in the software package MIRIAD. In this paper, I will use the approach of Hamaker et al. (1996) to develop a method for calibration of single dish data. This method is particularly useful for observations of pulsars. In the final section of the paper I apply the method to observations of the pulsar PSR J1359-6038 using the $20 \mathrm{~cm}$ multibeam receiver on the Parkes radio telescope and discuss the results.

\section{Representation}

Following Hamaker et al. (1996) I describe the propagation of an electromagnetic wave in the $x y z$ coordinate system by

$$
e_{2}=\left\langle\left(\begin{array}{l}
e_{x} \\
e_{y}
\end{array}\right)\right\rangle
$$

where the axis of propagation is the $z$ axis, and $e_{x}$ and $e_{y}$ are complex. The coherency properties of the electric field can be expressed in $x y$ coordinates by the coherency matrix (Born \& Wolf 1964)

$$
e=\left\langle\left(\begin{array}{l}
e_{x} e_{x}^{*} \\
e_{x} e_{y}^{*} \\
e_{y} e_{x}^{*} \\
e_{y} e_{y}^{*}
\end{array}\right)\right\rangle
$$

where the $*$ denotes the complex conjugate. The electromagnetic field is converted to electric voltage in the feed of a radio telescope which, in the case of linear feeds, consists of two input probes aligned along $x$ and $y$. A correlator multiplies and averages these voltages to produce the voltage vectors

$$
v_{2}=\left\langle\left(\begin{array}{c}
v_{x} \\
v_{y}
\end{array}\right)\right\rangle ; \quad v=\left\langle\left(\begin{array}{c}
v_{x} v_{x}^{*} \\
v_{x} v_{y}^{*} \\
v_{y} v_{x}^{*} \\
v_{y} v_{y}^{*}
\end{array}\right)\right\rangle .
$$

Generally, one is interested in the (true) Stokes parameters, $I, Q, U$, and $V$, which in combination form the Stokes vector $e^{S}$. One can express $e^{S}$ in terms of the electric field vector, $e$, by

$$
e^{S}=\left(\begin{array}{l}
I \\
Q \\
U \\
V
\end{array}\right)=T e ; \quad T=\left(\begin{array}{rrrr}
1 & 0 & 0 & 1 \\
1 & 0 & 0 & -1 \\
0 & 1 & 1 & 0 \\
0 & -i & i & 0
\end{array}\right)
$$

The propagation of the radiation from the astrophysical source of interest to the output from the correlator can be described in the following way (Hamaker et al. 1996). Let the effects of Faraday Rotation (both through the interstellar medium and the ionosphere) and parallactic angle rotation be combined into the matrix $R$. We let the matrix $F$ represent the feed response (including gains, phases, 
and leakage terms). Hence the voltages at the two probes can be described by

$$
v_{2}=F R
$$

and the correlator voltage vector is given by

$$
v=\left(F \otimes F^{*}\right)\left(R \otimes R^{*}\right) T^{-1} e^{S}
$$

where $\otimes$ denotes the Kronecker (or outer) product (Hamaker et al. 1996). The rotation matrix, $R$, and the feed response matrix, $F$, are given by Hamaker et al. (1996) as

$$
\begin{aligned}
& R=\left(\begin{array}{rr}
\cos \phi & -\sin \phi \\
\sin \phi & \cos \phi
\end{array}\right) ; \\
& F=\left(\begin{array}{rr}
G & B \\
-C & H
\end{array}\right)
\end{aligned}
$$

where each of $B, C, G$, and $H$ are complex terms. It is important to note that Hamaker et al. (1996) have shown that such a formalism does not involve any approximations, although often, as below, the matrices are then expanded only to first order.

In this formalism, however, unlike that of Britton (2000), there is no direct physical association of the variables $B, C, G$, and $H . B$ and $C$ can be thought of as 'leakage' terms; they describe the leakage of the opposite polarisation into either receptor. The real parts of $G$ and $H$ relate to the sensitivity of the two probes and the difference in the imaginary parts of $G$ and $H$ relates to the phase offset between the two channels (often called 'instrumental phase' for a pair of linear receptors). However, this is not strictly correct, as $G$ and $H$ also mixed with the leakage terms (Hamaker et al. 1996).

Expanding equation (6) and ignoring (for now) the effects of rotation we thus obtain

$$
\begin{aligned}
\left(\begin{array}{l}
v_{x x^{*}} \\
v_{x y^{*}} \\
v_{y x^{*}} \\
v_{y y^{*}}
\end{array}\right)= & \frac{1}{2}\left(\begin{array}{rrrr}
G G^{*} & G B^{*} & B G^{*} & B B^{*} \\
-G C^{*} & G H^{*} & -B C^{*} & B H^{*} \\
-C G^{*} & -C B^{*} & H G^{*} & H B^{*} \\
C C^{*} & -C H^{*} & -H C^{*} & H H^{*}
\end{array}\right) \\
& \times\left(\begin{array}{rrrr}
1 & 1 & 0 & 0 \\
0 & 0 & 1 & i \\
0 & 0 & 1 & -i \\
1 & -1 & 0 & 0
\end{array}\right)\left(\begin{array}{c}
I \\
Q \\
U \\
V
\end{array}\right)
\end{aligned}
$$

where the scaling factor $1 / 2$ arises from the determinant of $T$. Multiplying the two $4 \times 4$ matrices together we arrive at

\section{Measured Stokes Parameters}

Using equation (9) above and defining the measured Stokes parameters in the case of linear feeds by

$$
\begin{aligned}
I_{m} & =v_{x x^{*}}+v_{y y^{*}} \\
Q_{m} & =v_{x x^{*}}-v_{y y^{*}} \\
U_{m} & =v_{x y^{*}}+v_{y x^{*}} \\
i V_{m} & =v_{x y^{*}}-v_{y x^{*}}
\end{aligned}
$$

we can express the measured Stokes parameters in terms of the true Stokes parameters as follows:

$$
\begin{aligned}
I_{m}= & \frac{1}{2} I\left(g_{1}^{2}+g_{2}^{2}+h_{1}^{2}+h_{2}^{2}\right) \\
& +\frac{1}{2} Q\left(g_{1}^{2}+g_{2}^{2}-h_{1}^{2}-h_{2}^{2}\right) \\
& +U\left(g_{1} b_{1}+g_{2} b_{2}-h_{1} c_{1}-h_{2} c_{2}\right) \\
& +V\left(g_{1} b_{2}-g_{2} b_{1}+h_{1} c_{1}-h_{2} c_{2}\right) \\
Q_{m}= & \frac{1}{2} I\left(g_{1}^{2}+g_{2}^{2}-h_{1}^{2}-h_{2}^{2}\right) \\
& +\frac{1}{2} Q\left(g_{1}^{2}+g_{2}^{2}+h_{1}^{2}+h_{2}^{2}\right) \\
& +U\left(g_{1} b_{1}+g_{2} b_{2}+h_{1} c_{1}+h_{2} c_{2}\right) \\
& +V\left(g_{1} b_{2}-g_{2} b_{1}-h_{1} c_{2}+h_{2} c_{1}\right) \\
U_{m}= & I\left(h_{1} b_{1}+h_{2} b_{2}-g_{1} c_{1}-g_{2} c_{2}\right) \\
& +Q\left(-g_{1} c_{1}-g_{2} c_{2}-h_{1} b_{1}-h_{2} b_{2}\right) \\
& +U\left(g_{1} h_{1}+g_{2} h_{2}\right)+V\left(g_{1} h_{2}-g_{2} h_{1}\right) \\
V_{m}= & I\left(g_{1} c_{2}-g_{2} c_{1}+h_{1} b_{2}-h_{2} b_{1}\right) \\
& +Q\left(g_{1} c_{2}+g_{2} c_{1}-h_{1} b_{2}+h_{2} b_{1}\right) \\
& +U\left(-g_{1} h_{2}+g_{2} h_{1}\right)+V\left(g_{1} h_{1}+g_{2} h_{2}\right)
\end{aligned}
$$

where all second order terms in $B$ and $C$ have been omitted. Somewhat unconventionally I have defined each complex term above by, for example, $G=g_{1}+i g_{2}$ rather than $G=$ $g_{1} e^{i g_{2}}$ to simplify the notation. Note that these parameters are assumed to be time independent but will not, in general, be frequency independent. In a perfect system one has $b_{1}=b_{2}=0, c_{1}=c_{2}=0, g_{1}=h_{1}=1$, and $g_{2}=$ $h_{2}=0$, and hence the measured Stokes parameters are identically equal to the true Stokes parameters (ignoring the effects of rotation of Stokes $Q$ into $U$ along the line of sight).

If the gains and instrumental phase have been solved for by some other method, as is often the case (e.g. through observations of a polarised calibration signal injected directly into the feed), then $g_{1}=h_{1}=1$ and $g_{2}=h_{2}=0$

$$
\left(\begin{array}{l}
v_{x x^{*}} \\
v_{x y^{*}} \\
v_{y x^{*}} \\
v_{y y^{*}}
\end{array}\right)=\frac{1}{2}\left(\begin{array}{rrrr}
G G^{*}+B B^{*} & G G^{*}-B B^{*} & G B^{*}+B G^{*} & i\left(G B^{*}-B G^{*}\right) \\
-G C^{*}+B H^{*} & -G C^{*}-B H^{*} & G H^{*}-B C^{*} & i\left(G H^{*}+B C^{*}\right) \\
-C G^{*}+H B^{*} & -C G^{*}-H B^{*} & -C B^{*}+H G^{*} & -i\left(C B^{*}+H G^{*}\right) \\
C C^{*}+H H^{*} & C C^{*}-H H^{*} & -C H^{*}-H C^{*} & -i\left(C H^{*}-H C^{*}\right)
\end{array}\right)\left(\begin{array}{c}
I \\
Q \\
U \\
V
\end{array}\right) .
$$


and equation (11) simplifies to:

$$
\begin{gathered}
I_{m}=I+U\left(b_{1}-c_{1}\right)+V\left(b_{2}+c_{2}\right) \\
Q_{m}=Q+U\left(b_{1}+c_{1}\right)+V\left(b_{2}-c_{2}\right) \\
U_{m}=U+I\left(b_{1}-c_{1}\right)-Q\left(b_{1}+c_{1}\right) \\
V_{m}=V+I\left(b_{2}+c_{2}\right)-Q\left(b_{2}-c_{2}\right) .
\end{gathered}
$$

We also note, as Britton (2000) has done, that a relationship holds between the measured and true Stokes parameters via

$$
I_{m}^{2}-Q_{m}^{2}-U_{m}^{2}-V_{m}^{2}=K\left(I^{2}-Q^{2}-U^{2}-V^{2}\right)
$$

where $K$ is time invariant and is related to the determinant of the matrix in equation (11). This expression, the so-called invariant interval, is not frequency invariant however, as it depends on the (frequency dependent) terms in the $F$ matrix. Britton's idea of using the invariant interval for timing purposes has been used to great effect by van Straten et al. (2001).

\section{Effects of Feed Rotation}

It is sometimes stated that performing a (short) observation, rotating the feed through $90^{\circ}$ and observing again and summing the two observations has the effect of cancelling the effect of the leakage parameters. However, this is not the case, even to first order. The effect of feed rotation effectively converts $Q$ to $-Q$ and $U$ to $-U$. To sum the two observations one essentially averages the sum of the two values of $I_{m}$ and $V_{m}$ and averages the differences of the two $Q_{m}$ and $U_{m}$ measurements. Assuming the gains are correctly calibrated, then using equation (12) above, one obtains:

$$
\begin{aligned}
I_{m} & =I+V\left(b_{2}+c_{2}\right) \\
Q_{m} & =Q+U\left(b_{1}+c_{1}\right) \\
U_{m} & =U-Q\left(b_{1}+c_{1}\right) \\
V_{m} & =V+I\left(b_{2}+c_{2}\right)
\end{aligned}
$$

and the total linear polarisation is thus

$$
L_{m}=L \sqrt{1+\left(b_{1}+c_{1}\right)^{2}} .
$$

These last two equations show that the measured Stokes parameters are now a better approximation to the true Stokes parameters than a single observation would yield. However, even though the errors in $I_{m}$ and $L_{m}$ are likely to be small, the error in $V_{m}$ can potentially be large if $V$ is small and $b_{2}+c_{2}$ is significant. The error in the position angle of the linear polarisation will be of order $\tan ^{-1}\left(b_{1}+c_{1}\right)$.

A further potentially interesting measurement is to subtract $V_{m}$ from one observation with that from an observation where the feed is rotated through $90^{\circ}$. In this case one obtains

$$
V_{m}=-Q\left(b_{2}-c_{2}\right) .
$$

If $Q$ is large, one can then obtain $b_{2}-c_{2}$ directly.

\section{Effects of Observing a Polarised Calibration Signal}

It is often the case that a cal probe is included in the receiver package. This probe usually injects a signal between the two (linear) probes at an angle of $45^{\circ}$ to both. The cal thus has $I=U$ and $Q=V=0$. Imagine now that the two probes have identical gains and there is no instrumental rotation (i.e. we can set $g_{1}=h_{1}=1$ and $g_{2}=h_{2}=0$ ). Then, if the leakage terms are present, observations of the cal will yield a signal

$$
\begin{aligned}
v_{x x^{*}} & =\frac{1}{2} U\left(1+2 b_{1}\right) \\
v_{y y^{*}} & =\frac{1}{2} U\left(1-2 c_{1}\right) \\
V_{m} & =U\left(b_{2}+c_{2}\right) .
\end{aligned}
$$

The first two expressions look like gain difference between the two probes, and the presence of $V$ looks like instrumental rotation of $U$ into $V$. Hence, even though we started with a system with perfect gains and phases, we derive using equation (12)

$$
\begin{aligned}
& g_{1}=\frac{1}{1+2 b_{1}} \\
& h_{1}=\frac{1}{1-2 c_{1}} \\
& h_{2}=b_{2}+c_{2}
\end{aligned}
$$

and hence the system looks impure! One then re-observes the cal (or any other pure U signal) with these terms in equation (11) and one obtains

$$
\begin{aligned}
I_{m} & =U\left(1-\frac{b_{1}}{\left(1+2 b_{1}\right)^{2}}+\frac{c_{1}}{\left(1+2 c_{1}\right)^{2}}+\frac{1}{2} b_{2}^{2}-\frac{1}{2} c_{2}^{2}\right) \\
Q_{m} & =U\left(\frac{-b_{1}}{\left(1+2 b_{1}\right)^{2}}+\frac{c_{1}}{\left(1+2 c_{1}\right)^{2}}+\frac{1}{2} b_{2}^{2}-\frac{1}{2} c_{2}^{2}\right) \\
U_{m} & =U\left(\frac{b_{1}+2 b_{1}^{2}-c_{1}+2 c_{1}^{2}+1}{\left(1+2 b_{1}\right)\left(1-2 c_{1}\right)}+b_{2}\left(b_{2}+c_{2}\right)\right) \\
V_{m} & =U\left(\frac{2 b_{2}\left(c_{1}+b_{1}\right)}{\left(1+2 b_{1}\right)\left(1-2 c_{1}\right)}-b_{1}\left(b_{2}+c_{2}\right)\right) .
\end{aligned}
$$

This implies that one does not, in general, measure a pure $U$ signal in spite of the calibration procedure.

\section{Circular Feeds}

The Parkes radio telescope has receivers with linear feeds. However, many other telescopes have circular feed systems. To apply this method to receivers with circular feeds one needs to change the Stokes vector $e^{S}$ to

$$
e^{S}=\left(\begin{array}{c}
I \\
V \\
Q \\
U
\end{array}\right)
$$


and equation (10) to read

$$
\begin{aligned}
I_{m} & =v_{r r^{*}}+v_{l l^{*}} \\
V_{m} & =v_{r r^{*}}-v_{l l^{*}} \\
Q_{m} & =v_{r l^{*}}+v_{l r^{*}} \\
i U_{m} & =v_{r l^{*}}-v_{l r^{*}} .
\end{aligned}
$$

The algebra can then be worked through to obtain expressions for the measured Stokes parameters in terms of the true parameters as in equation (11).

\section{Application}

From equation (11) it can be seen there are 4 measured quantities (the Stokes parameters) and a total of 12 unknowns. These 12 unknowns comprise the 4 leakage parameters $\left(b_{1}, b_{2}, c_{1}, c_{2}\right)$, the 4 gain terms $\left(g_{1}, g_{2}\right.$, $\left.h_{1}, h_{2}\right)$, the total intensity, linear and circular polarisation of the source, and an unknown angle $\phi$ which deals with rotation along the line of sight such that $Q=L \cos \phi$ and $U=L \sin \phi$. This angle is made up of the intrinsic position angle of the source, the rotation measure through the interstellar medium and ionosphere, the parallactic angle and the feed angle(s) with respect to some reference frame. Of these angles, only the ionospheric rotation measure and the parallactic angle are time variable. Unless some independent measurement can be made of the ionosphere, this introduces unwanted noise into the calibration matrices. Ignoring this, rotating the feed relative to the sky (for example as naturally occurs with a feed mounted on an alt-az telescope) can be used to determine the 12 unknowns. Provided we have sufficient independent measurements of the Stokes parameters at a variety of parallactic angles, we can, in principle, determine all the unknowns. Sault et al. (1996) show that observations of an unpolarised source will yield only 9 of the unknowns, but that observations of a strongly linearly polarised source can determine all 12 .

Pulsars make ideal sources for (self-)calibration as they are generally highly linearly polarised and have a reasonable degree of circular polarisation. Furthermore, the polarisation changes across the pulse profile and one can thus use the information in multiple phase bins at a given pointing to help solve for the unknowns. The main drawback to using pulsars as calibrators is that they scintillate which implies that the total intensity, $I$, can vary significantly on timescales of order minutes. The fractional polarisation is not affected by scintillation, however, and therefore to overcome this problem one is forced to normalise the measured Stokes parameters by $I_{m}$ at the expense of being unable to measure the true value of I. Unfortunately this procedure introduces errors as, of course, $I_{m}$ is not equal to $I$. In practice these errors will be small unless the source is highly polarised and $b_{1}-c_{1}$ is large.

A previous method for calibration of pulsar signals was given in the Appendix of Stinebring et al. (1984)

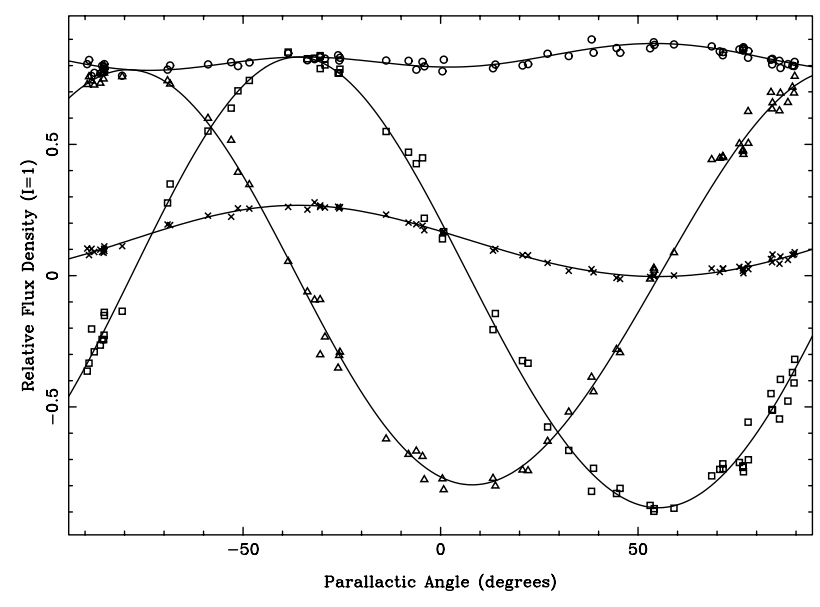

Figure 1 Measured Stokes parameters for PSR J1359-6038 as a function of parallactic angle. Here, $L_{m}$ is denoted by circles, $Q_{m}$ by squares, $U_{m}$ by triangles, and $V_{m}$ by crosses. The lines denote the best fit to the data after solving equation (11).

and is still in use (e.g. Weisberg et al. 1999). However, their method involves a number of simplifications and assumptions which, in my view, are incorrect in some cases and which are, in any case, no longer necessary to make. Any least squares fitting algorithm can easily solve for 12 unknowns with $\sim 100$ data points in a matter of seconds and the full equations should thus be used.

Data on PSR J1359-6038 were collected on two separate occasions in July and Novmeber 2000. Each observing session lasted approximately 10 days and we obtained 65 and 66 independent observations covering the whole parallactic angle range available (approximately $\pm 94^{\circ}$ ). These data were taken using the $64 \mathrm{~m}$ Parkes telescope using the centre beam of the multibeam receiver at a central observing frequency of $1318 \mathrm{MHz}$ and with a total bandwidth of $128 \mathrm{MHz}$. At the start of the observing session observations were made of the flux calibrator Hydra A to obtain the system equivalent flux density. Then, a $90 \mathrm{sec}$ observation of the cal signal was made followed immediately by a 3 min observation of the pulsar. The pulsar observation was calibrated for differential gain and phase in the two probes based on the results from the cal observation and flux calibrated from the observations of Hydra A. Pulse profiles were formed in each of $I_{m}, Q_{m}, U_{m}$, and $V_{m}$ with 256 phase-bins per profile and 8 frequency channels across the $128 \mathrm{MHz}$ total bandwidth. $Q_{m}, U_{m}$, and $V_{m}$ were then normalised by $I_{m}$.

Figure 1 shows the measured (normalised) Stokes parameters as a function of parallatic angle from data taken in November 2000 from the peak of the pulse profile in one of the frequency channels centred at $1365 \mathrm{MHz}$. It can clearly be seen that the circular polarisation, $V_{m}$, varies significantly with parallactic angle and that the variations are in phase with the variations in $Q_{m}$. The deviation in $V_{m}$ is very large with a peak-to-peak amplitude of about 0.2 . The implication of this (from equation (12)) is that $b_{2}-c_{2}$ is of this order, given that $Q_{m}$ is about unity. 


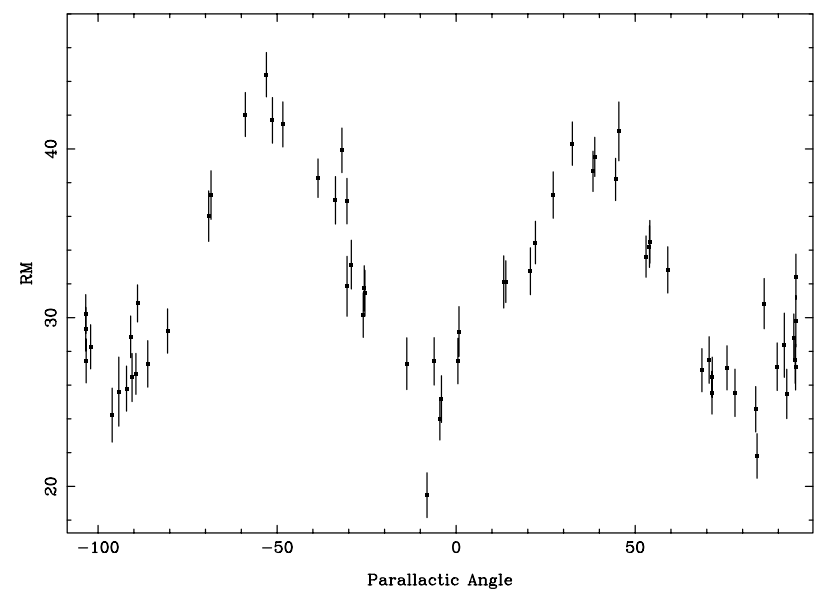

Figure 2 Rotation measure of PSR J1359-6038 as a function of parallactic angle before polarisation calibration. The error bars are formal errors from the fitting process used to determine the rotation measure.

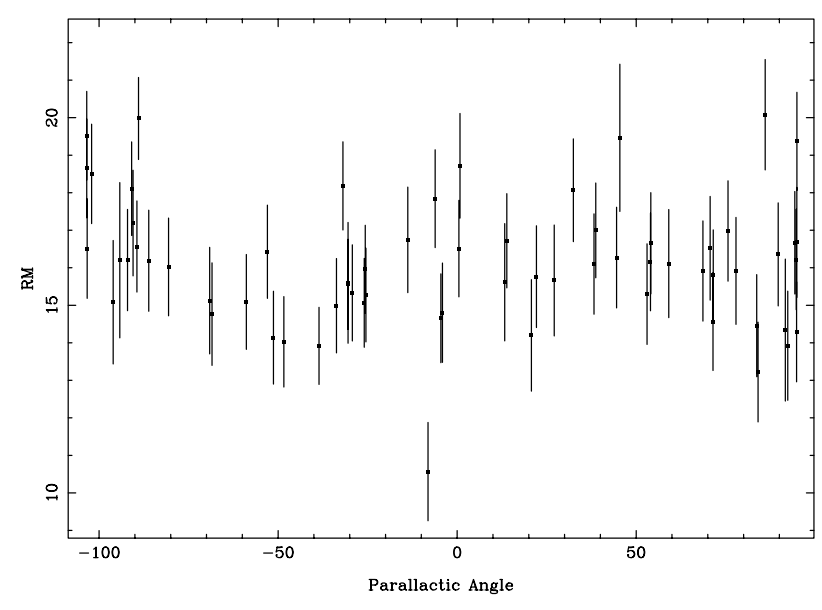

Figure 3 Rotation measure of PSR J1359-6038 after polarisation calibration.

It was shown in Section 5 that observations of the cal induces subsequent errors in the observed Stokes parameters. These errors can be removed by (re-)solving for the gains and phase terms (although nominally the cal observations are used to set these) using equation (11). Practically, however, I set $g_{1}=1$ and $h_{1}=0$ and leave them fixed; $g_{2}$ and $h_{2}$ then measure the relative gain and phase differences between the two probes. I then used a least squares fitting algorithm (in this case the LevenbergMaquart Method; LMM) to minimise the residuals of $\left(\left|Q_{m}-Q\right|+\left|U_{m}-U\right|+\left|V_{m}-V\right|\right)$ and solve for the 9 unknowns from equation (11) given the data (remembering that $I_{m}$ is fixed at 1 , all the observed Stokes parameters are normalised by $I_{m}$ and $g_{1}=1, h_{1}=0$ ). The result of the fitting is shown in Figure 1. At this particular frequency, $b_{1}=0.0, b_{2}=-0.05, c_{1}=0.02$, and $c_{2}=0.12$. Given this and the discussion in Section 4, rotating the feed through $90^{\circ}$ and observing again still induces a leakage of $I$ into $V$ of order $7 \%$, about the same size as the true $V$ signal in many pulsars. As pointed out, an independent measurement of $b_{2}-c_{2}$ can be obtained in this way, and confirms the measurements derived above.

At this point, all the parameters of the matrix form of equation (11) have effectively been determined. The true Stokes parameters can then be obtained by multiplying the observed Stokes parameters with the inversion of this matrix. This can be achieved with, for example, the numerical recipes routine LUBKSB.

Figure 2 shows the rotation measure of the pulsar as a function of parallactic angle prior to polarisation calibration. There are clear systematics in the data with sinusoidal variations of $\sim 10 \mathrm{rad} \mathrm{m}^{-2}$ about a mean of $35 \mathrm{rad} \mathrm{m}^{-2}$. These are due to the frequency dependency of the leakage terms $B$ and $C$. Figure 3 shows the effects of the calibration. The mean rotation measure is now $16 \mathrm{rad} \mathrm{m}^{-2}$ and the systematic effects are largely absent.

\section{Conclusions}

Using the excellent Hamaker et al. (1996) paper as a mathematical foundation, I have derived the matrices linking the true Stokes parameters to the observed Stokes parameters for single dish observations. It is then a computationally trivial task to solve for the terms in this matrix by least squares minimisation and thus provide a polarisation calibration of the feed and receiver system.

\section{Acknowledgments}

I would like to thank M. Kesteven for fruitful discussions and a careful reading of this manuscript, and R. Manchester for discussion on the calibration procedure used at Parkes. The referee, M. Britton, made a valuable contribution to improving the paper. A. Karastergiou and L. Nicastro provided assistance with the observations.

\section{References}

Born, M., \& Wolf, E. 1964, Principles of Optics (Pergamon Press) Britton, M. 2000, ApJ, 532, 1240

Hamaker, J. P., Bregman, J. D., \& Sault, R. J. 1996, A\&ASS, 117, 137

Sault, R. J., Hamaker, J. P., \& Bregman, J. D. 1996, A\&ASS, 117, 149 Stinebring, D. R., Cordes, J. M., Rankin, J. M., Weisberg, J. M., \& Boriakoff, V. 1984, ApJSS, 55, 247

van Straten, W., Bailes, M., Britton, M., Kulkarni, S. R., Anderson,

S. B., Manchester, R. N., Sarkissian, J. 2001, Nature, 452, 158 Weisberg, J. M., et al. 1999, ApJSS, 121, 171 